\title{
Generating System Wellbeing Index Evaluation Using Genetic Algorithm
}

\author{
Ahmed S. Al-Abdulwahab \\ Electrical and Computer Engineering Department, \\ Faculty of Engineering, King Abdulaziz University, \\ P.O.Box 80204, Jeddah 21589, Saudi Arabia
}

\begin{abstract}
Reliability assessment of generation system is a crucial task used to be done using deterministic approaches. However, due to the practical limitations of these approaches, they have been gradually replaced by probabilistic techniques. Nevertheless, there is a considerable reluctance in many electric power utilities to completely abandon deterministic considerations. To fulfill the industry need, wellbeing analysis has been developed to combine the deterministic and the probabilistic approaches in a single framework. Analytical techniques or Monte Carlo Simulation have been used to evaluate wellbeing indices. However, analytical approaches are complicated and mathematically demanding and simulation technique requires a huge amount of computing time, and large memory size. This still prevents the utilities to benefit from the wellbeing framework. This paper presents a novel Genetic Algorithm (GA) based technique to calculate the wellbeing indices. Hopefully, this will encourage the industry to benefit from the wellbeing analysis. The features of the GA are utilized to collect and identify the health, marginal and at risk wellbeing states and to calculate the associated wellbeing indices. The proposed technique is applied to the IEEE-RBTS and the resulting wellbeing indices are compared to those obtained using a conventional analytical technique. The results show that the outcome of both techniques is virtually identical. The effect of the GA parameters on the wellbeing indices is examined. The proposed GA based technique in the manner applied in this study is simple, practical and valid to calculate the wellbeing indices.
\end{abstract}




\section{Introduction}

Conventional generating capacity adequacy assessment over the past several decades has gradually evolved from a deterministic appraisal to one utilizing probabilistic concepts. A wide range of probabilistic techniques have been employed to incorporate risk in the evaluation ${ }^{[1-4]}$. There is, however, considerable appeal in utilizing deterministic techniques rather than more complicated probabilistic methodologies. Wellbeing analysis has been developed to combine the deterministic and the probabilistic approaches in a single framework ${ }^{[5-7]}$.

System wellbeing analysis utilizes three wellbeing indices; the probability of health $\mathrm{P}(\mathrm{H})$, the probability of margin $\mathrm{P}(\mathrm{M})$ and the probability of risk $\mathrm{P}(\mathrm{R})$. These probabilities reflect the three states that the system can reside in. The probability of health $\mathrm{P}(\mathrm{H})$ is the probability of the system being in the healthy state where the available reserve is equal to or greater than the required capacity reserve. In this paper, the capacity of the largest unit (CLU) is used as the capacity reserve criterion. The probability of margin $\mathrm{P}(\mathrm{M})$ is the probability of the system being in the marginal state. In this state, the available reserve is less than the required capacity reserve but greater than zero. The probability of risk $\mathrm{P}(\mathrm{R})$ is the probability of the system being in the at risk state. In this state, the load exceeds the available generation.

Analytical techniques or Monte Carlo Simulation (MCS) are conventionally used to evaluate wellbeing indices ${ }^{[8]}$. The two basic analytical techniques used are the Contingency Enumeration (CE) method and the Conditional Probability Capacity Outage Probability Table approach (CPCOPT) ${ }^{[6,9]}$.

In the CE approach, a generation model is created which includes all possible combinations of the existing generating unit outages with their corresponding probabilities. The available reserve in each state is compared with the CLU in that state. Then the system states are categorized to the three possible wellbeing states ${ }^{[9,10]}$. The CE approach is impractical to be used for even a relatively small system as all system states need to be considered and evaluated. The CPCOPT approach has been developed to overcome the limitations of the CE approach ${ }^{[6]}$. There are three steps in this technique. The first step is to determine the LOLP, which is the probability of risk, using the well-known LOLP method ${ }^{[1]}$. The second step is to obtain the probability of health using a similar 
technique in which several COPT are created and convolved with the load model. In each case, the $\mathrm{P}(\mathrm{H})$ is obtained and weighted with the corresponding generating unit probabilities. In the final step, the $\mathrm{P}(\mathrm{H})$ and the $\mathrm{P}(\mathrm{R})$ are subtracted from 1 to evaluate the probability of margin $[6,10,11]$. Although, the CPCOPT overcomes the limitations of the CE approach, it is still complicated and mathematically demanding ${ }^{[8]}$. The utilization of MCS for wellbeing indices evaluation is introduced to overcome the complexity of analytical techniques ${ }^{[8]}$. MCS estimates the wellbeing indices by simulating the actual process and random behavior of the system. However, MCS requires a huge amount of computing time, and large memory size ${ }^{[12]}$. This suggests further research to develop more practical yet simple technique to deal with the wellbeing indices calculations. Surprisingly, the GA has never been used to calculate the wellbeing indices. This paper presents a new GA based technique to calculate the wellbeing indices for power generation systems. The GA is an optimization and search technique that does not require mathematical modeling due to the fact that it is based on random but controlled process. The features of the GA are utilized to identify the health, marginal and at risk wellbeing states and to calculate the associated wellbeing indices.

The paper is organized as follows: Section 2 presents the proposed GA based technique and formulates the model. Section 3 presents the application of the proposed technique to the IEEE-RBTS. Section 4 elaborates on the sensitivity of the proposed technique to the GA parameters. Concluding remarks are drawn in Section 5.

\section{Wellbeing Analysis Using GA Search Method}

The GA has emerged as a powerful search tool used for search and optimization in power system analysis and applications ${ }^{[13-20]}$. References [15] and [16] introduce the utilization of the GA as a powerful tool for the conventional and traditional reliability indices evaluation. This paper presents the GA as a tool to calculate the wellbeing indices. The GA is used as a search tool to identify the system states. It is random yet controlled process that generates system states. These states are represented as chromosomes and the fit individuals are used. Each system state is classified as healthy, marginal or at risk state. The proposed GA based technique is summarized in the following section. 


\subsection{Description of the Proposed Technique}

1) An initial population is generated randomly. This population contains individual chromosomes. Each chromosome represents a system state which is encoded as a binary string with length equal to the number of generating units. Each unit is represented by one bit with a value of ' 1 ' or ' 0 ' corresponding to 'in service' or 'out of service' states.

2) The fitness of each individual (system state) is evaluated by calculating its probability. It is typical to have some repeated system states because it was generated randomly. The fitness of these repeated states is set to a very small value in the order of $10^{-8}$. Thus, only the unrepeated states are considered and added to a system states array.

3) Selection operation can be performed using different techniques such as Roulette selection, tournament selection and other techniques. Roulette wheel selection has two major disadvantages. First of all, it can handle only maximization problems, secondly, scaling of the fitness becomes very important near convergence. Members with very poor fitness die out very early causing premature convergence. Therefore, the tournament selection technique is used in this research work to select the fit strings (system states). It overcomes the disadvantages of the Roulette selection technique and one of the most used approaches. The system state probability is used as the fitness function. The states that have higher probability have more chances to be selected. The population members are randomly divided into subgroups and members with the best fitness among the subgroups get selected for reproduction. This process decreases the chances of generating system states with low probability of occurrence. Therefore, the selection process acts as the truncation technique.

4) The crossover operation follows the selection process. A single point crossover is used in this research. However, multi-point crossover can be used and explored for this kind of applications. The newly selected strings are paired together at random. Then, an integer position along every pair of strings is selected uniformly at random. Finally, based on a probability of crossover, the paired strings undergo crossing over at the integer position along the string. This results in new pairs of strings (system states).

5) Mutation operation follows the previous two GA operation to finalize the reproduction process. An occasional random alteration of a 
string based on probability of mutation is performed. This involves changing a 1 to a 0 and vice versa.

6) At this stage, the new generation is manifested. The total probability of the collected states in the system states array is calculated to keep track of the progress in collecting the fit system states,. This probability is designated as cumulative probability.

7) The wellbeing indices are calculated for the recovered states.

8) Different stopping criteria can be used. A maximum number of generations (iterations) can be used as a stopping criterion. Another stopping criterion is stopping the reproduction operation when there is no improvement (increase) in the cumulative probability of the collected states. Also, the regeneration can be terminated after finding a desired maximum cumulative probability. The last two alternatives are not suitable for this kind of application due to the fact that the cumulative probability and the other wellbeing indices are too low. Therefore, the incremental changes in the values of these quantities cannot be detected easily. Thus, a specified maximum number of generations is used a stopping criterion in this research work.

\subsection{Formulation of the Proposed Technique}

The probability of the units to be found in the 'in service' or 'out of service' states is calculated using Equation 1.

$$
\text { prob }_{j}=\left\{\begin{array}{c}
1-F O R_{j} \text { if } \text { state }_{i j}=1 \\
F \text { OR } R_{j} \text { if } \text { state }_{i j}=0
\end{array}\right.
$$

where,

state $_{i j}$ is the state of unit $j$ in system state $i$,

prob $_{j}$ is the state probability of unit $j$,

$F O R_{j}$ is the Forced Outage Rate of unit $j$

1) The fitness of each individual (system state) is evaluated by calculating its probability. State probability (fitness function) is calculated using Equation 2.

$$
\text { State } \text { prob }_{i}=\prod_{j=1}^{N} \text { prob }_{j}
$$

Where:

$N$ is the number of generating units 
2) For each system state in the system state array, the unit capacity is calculated using Equation 3.

$$
{\text { UnitStateC } a p_{i j}}=\text { State }_{i j} * \operatorname{Cap}_{j} \quad[\mathrm{MW}]
$$

Where:

UnitStateCap $p_{i j}$ is the capacity of the generating unit $j$ in system state $i$.

$\mathrm{Cap}_{j}$ is the capacity of unit ${ }_{j}$ in MW,

3) The CLU for each system state in the system states array is calculated using Equation 4.

$$
C L U_{i}=\operatorname{Max}\left({\text { UnitStateC } a p_{i j}}\right) \text { for } j \in[1 N] \quad[M W]
$$

4) The capacity in service is calculated for each system state recovered in the system states array for the current population using Equation 5.

$$
\text { State Cap } \text { C }_{i=1}^{N} \text { State }_{i j} * \text { Cap }_{j} \quad[M W]
$$

5) The available reserve margin for each system state for the states recovered in the system state array is calculated using Equation 6.

$$
\text { State Re } \text { Serve }_{i}=\text { State Cap }_{i}-P L \quad[M W]
$$

Where:

$P L$ is the system peak load in MW

6) The status of the system states recovered in the system state array is categorized to health, marginal or at risk state using Equation 7.

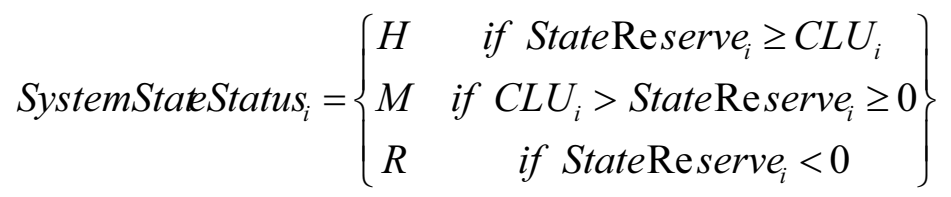

Where,

$H$ indicates that the $i_{\text {th }}$ system state is a healthy state

$M$ indicates that the $i_{\text {th }}$ system state is a marginal state

$R$ indicates that the $i_{\text {th }}$ system state is at risk state

7) At the end of each generation, the cumulative probability of the collected states is calculated using Equation 8.

$$
\text { Cprob }_{k}=\text { Cprob }_{k-1}+\sum_{i=1}^{M} \text { State }_{\text {prob }}
$$


where,

$\mathrm{Cprob}_{k}$ is the cumulative probability

$M$ is the total number of states collected in the $k_{t h}$ generation.

The cumulative probability for the initial population when $k$ equals zero is zero and it approaches 1.0 as reproduction iterations proceeds.

8) The wellbeing indices, $P H_{k}, P M_{k}$ and $P R_{k}$ are calculated for the recovered states in the $\mathrm{k}_{\text {th }}$ generation using Equations 9, 10 and 11.

$$
\begin{aligned}
& \mathrm{PH}_{k}=\mathrm{PH}_{k-1}+\text { State }_{\text {prob }} \text { if SystemStateStatuse }{ }_{i}=\mathrm{H} \\
& P M_{k}=P M_{k-1}+\text { State } \text { prob }_{i} \quad \text { if SystemStateStatuse } \text { St }_{i}=M \\
& P R_{k}=P R_{k-1}+\text { State }_{\text {prob }} \quad \text { if SystemStateStatuse } \text { S }_{i}=\mathrm{R}
\end{aligned}
$$

\section{Case Study}

The developed technique is applied to the IEEE-RBTS ${ }^{[21]}$. The IEEE-RBTS is an educational test system that has 11 generating units, ranged from $5 \mathrm{MW}$ to $40 \mathrm{MW}$ with a system peak load of $185 \mathrm{MW}$. Figure 1 shows the IEEE-RBTS. Two basic studies are simulated. Example 1 considers a population size of 300 and a number of generation of 100. Example 2 considers a population size of 100 and a number of generation of 500.A constant load model is used in this paper to calculate the annualized wellbeing indices. Table 1 shows the annualized wellbeing indices obtained using the proposed technique and the results obtained using the analytical CPCOPT technique presented in References ${ }^{[10]}$ and ${ }^{[11]}$ referred to as the "exact" results.

It can be seen from Table (1) that in both examples the error between the obtained indices using the proposed technique and the exact value is insignificant except for the $\mathrm{P}(\mathrm{R})$ calculated using Equation 11. The $\mathrm{P}(\mathrm{R})$ obtained using the GA technique is not accurate compared to the other two indices. This is due to that fact that many risk states are not recovered by the GA search technique because they have relatively small probabilities (fitness function) compared to the $\mathrm{P}(\mathrm{H})$ and $\mathrm{P}(\mathrm{M})$. To overcome this problem Equation 12 is used to calculate $P(R)$ instead of Equation 11.

$$
P R_{k}=1-P H_{k}-P M_{k}
$$




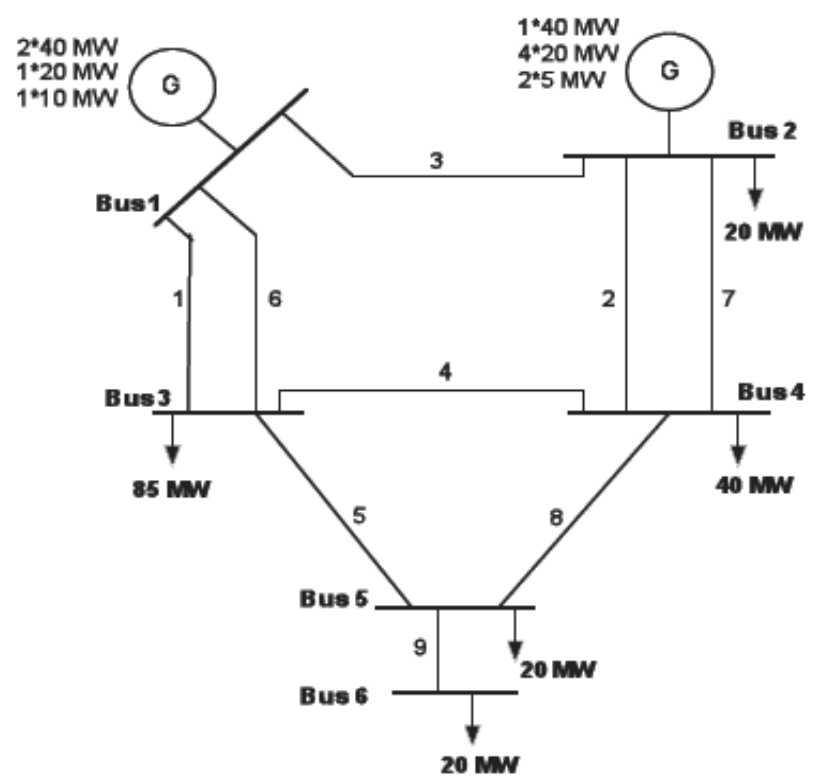

Fig. 1. The IEEE-RBTS.

Table 1. Annualized wellbeing indices.

\begin{tabular}{c|ccccc}
\multicolumn{2}{r}{} & \multicolumn{2}{c}{ Example 1 } & \multicolumn{2}{c}{ Example 2 } \\
\cline { 2 - 6 } & Exact & $\begin{array}{c}\text { Proposed } \\
\text { technique }\end{array}$ & $\begin{array}{c}\text { Error } \\
(\mathbf{\%})\end{array}$ & $\begin{array}{c}\text { Proposed } \\
\text { technique }\end{array}$ & $\begin{array}{c}\text { Error } \\
\text { (\%) }\end{array}$ \\
\cline { 2 - 6 } P(H) & 0.84628806 & 0.84628806 & 0.00000 & 0.84628806 & 0.00000 \\
P(M) & 0.14537033 & 0.14537023 & 0.00007 & 0.14536702 & 0.00004 \\
P(R)_Eq. 11 & 0.00834161 & 0.00830791 & 0.40400 & 0.00831225 & 0.35197 \\
P(R)_Eq. 12 & 0.00834161 & 0.00834171 & 0.00120 & 0.00834166 & 0.00059
\end{tabular}

This modification has improved the calculated $\mathrm{P}(\mathrm{R})$ as the error has been reduced from $0.4 \%$ to $0.0012 \%$.

The results shown in Table 1 show that the wellbeing indices have been acquired with great accuracy using the GA based technique. Moreover, neither complicated modeling nor mathematically demanding procedures were needed to reach this accuracy which is the case with analytical approaches. This is a great advantage for the GA based technique compared to the analytical techniques.

A basic evaluation of the wellbeing indices for the generation facilities using a constant load model is presented above. This technique 
can be extended to incorporate the variable load as well. A multiple steps load representation can be used and the same process explained above is implemented for each load step. Then the resulting indices are weighted by the probability of each load step. Finally, the weighted indices are aggregated to yield the annual indices.

\section{Sensitivity Analysis}

The effect of the GA parameters on the proposed technique is examined. These parameters include the generation size, population size, crossover probability and mutation probability. The three wellbeing indices, $\mathrm{P}(\mathrm{H}), \mathrm{P}(\mathrm{M})$ and $\mathrm{P}(\mathrm{R})$ are tracked.

The effect of different number of generations with a population size of 100 is considered in this case. Figure (2) shows the $\mathrm{P}(\mathrm{H}), \mathrm{P}(\mathrm{M})$ and $\mathrm{P}(\mathrm{R})$ obtained with the different number of generations. It can be seen from Fig. 2(a) that the proposed technique yields the exact value with as small as 10 number of generations. The same value is obtained with a higher number of generations.

Figure 2(b) shows the variation of the probability of $\mathrm{P}(\mathrm{M})$ with the different number of generations. It can be seen that the $\mathrm{P}(\mathrm{M})$ reaches the exact value with a higher number of generations unlike the case with the $\mathrm{P}(\mathrm{H})$ in which the exact value was obtained with a low number of generation. This is due to the fact that the $\mathrm{P}(\mathrm{H})$ is relatively high (close to 1.0) and the GA can easily recover the states with high probabilities. This results with recovering all healthy states which yield the exact value with only 10 generations. $\mathrm{P}(\mathrm{M})$ needed more number of generations to recover the marginal states.

Figure 2(c) shows the $\mathrm{P}(\mathrm{R})$ obtained using Equations 11 and 12. Using Equation 11 did not yield the correct $P(R)$ values even with as high as a number of generation of 500. This is because of the significantly low probabilities of the risk states. As explained earlier, Equation 12 is used which gives more accurate value compared to the exact value as shown in Fig. 2(c).

It is worth noting from Fig. 2(b and c) that lower $\mathrm{P}(\mathrm{M})$ and $\mathrm{P}(\mathrm{R})$ are obtained with a higher number of generation in some of the cases considered. This is due to the fact that the GA is a random search technique that may not generate some of the significant system states if 
the number of generation is not high enough. However, with the continuous increase in the number of generations, the $\mathrm{P}(\mathrm{M})$ and $\mathrm{P}(\mathrm{R})$ became more stable and with increasing pattern. Therefore, higher confident in the results can be achieved with higher number of generation.
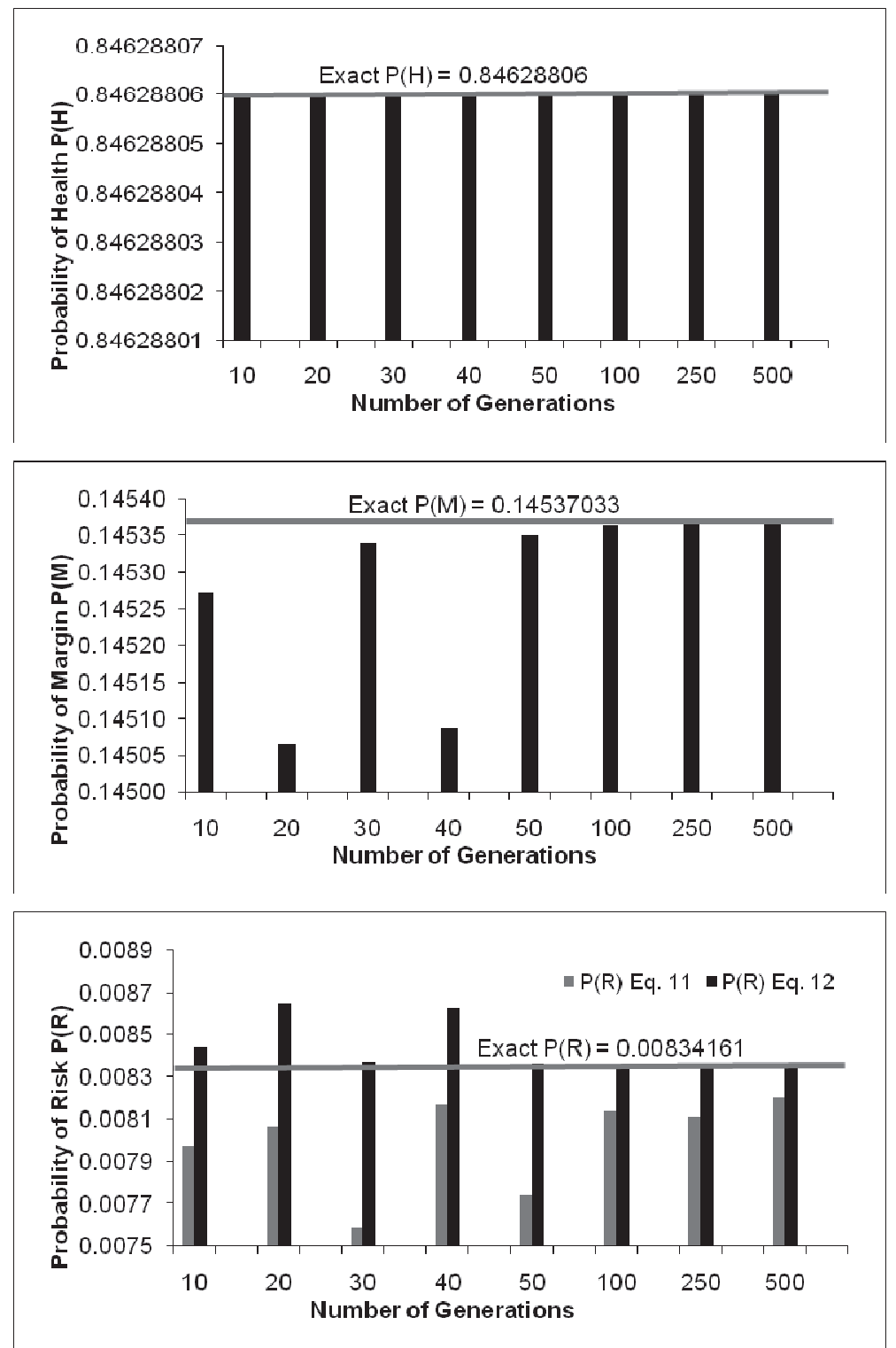

Fig. 2. Wellbeing indices with different number of generations and population size of 100. 
The effect of different population sizes with a number of generation of 50 is considered in this case. Figure 3 shows the $\mathrm{P}(\mathrm{H}), \mathrm{P}(\mathrm{M})$ and $\mathrm{P}(\mathrm{R})$ obtained with the different population sizes. Figure 3(a) shows that the proposed technique yields the exact value with a population size as small as 50 . The same value was obtained with higher population sizes.
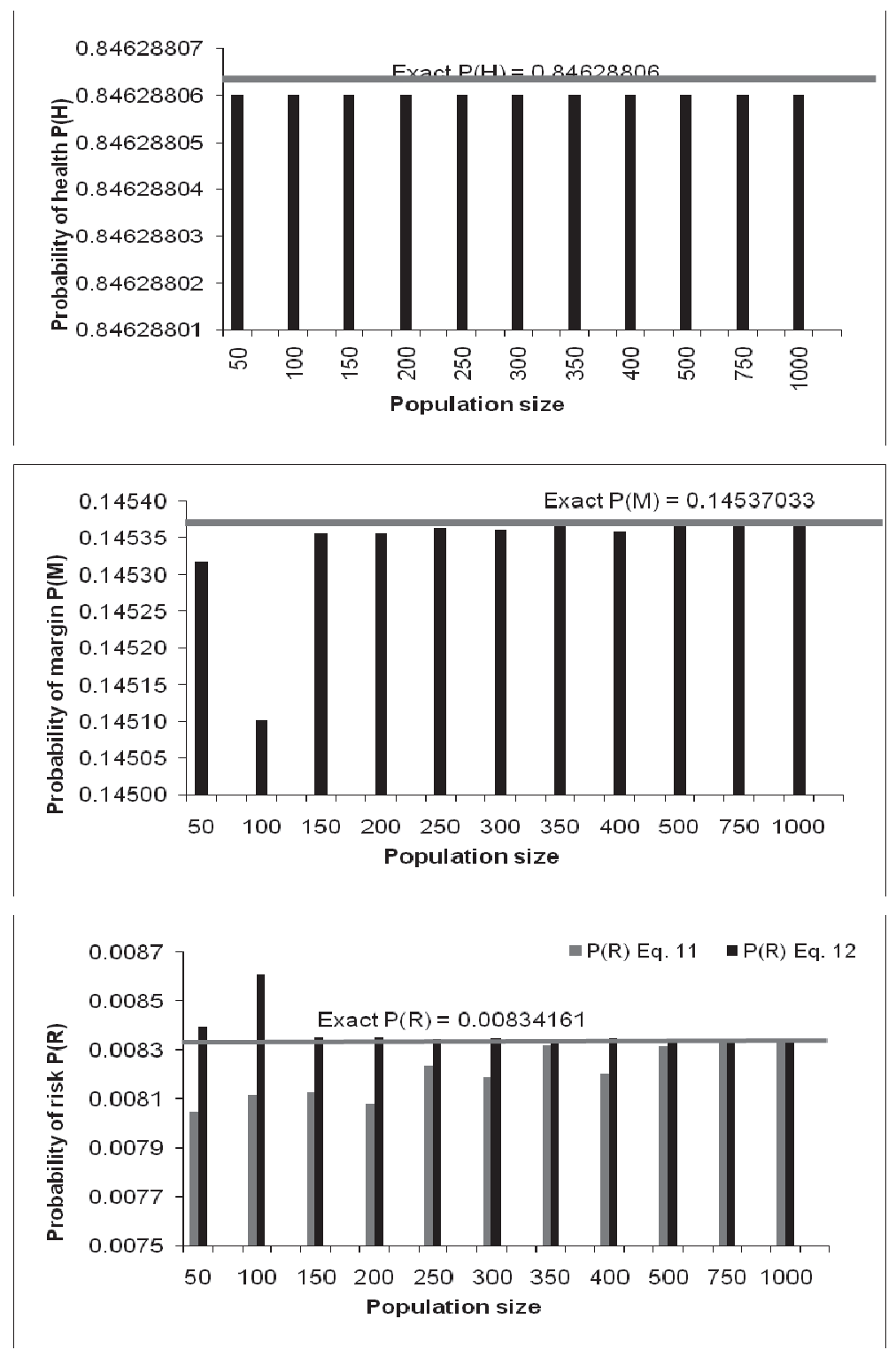

Fig. 3. Wellbeing indices with different population sizes and generation size of 50. 
Figure 3(b) shows the variation of the probability of $\mathrm{P}(\mathrm{M})$ with the different population sizes. It can be seen that the $\mathrm{P}(\mathrm{M})$ reaches the exact value with higher population sizes unlike the case with the $\mathrm{P}(\mathrm{H})$ in which the exact value was obtained with low population sizes.

Figure 3(c) shows the $\mathrm{P}(\mathrm{R})$ obtained using Equations 11 and 12. Both equations yield very accurate results compared to the exact value with high population size of 1000 . However, the result obtained with Equation 12 outperformed the result obtained with Equation 11 as Equation 12 gives very accurate results with low population sizes.

Figures 3(b and $c$ ) show that in some cases, lower $\mathrm{P}(\mathrm{M})$ and $\mathrm{P}(\mathrm{R})$ are obtained with higher number of population. This is due to the fact that the GA is a random search technique that may not generate some of the significant system states if number of populations is not high enough. However, with the continuous increase in the number of population, the $\mathrm{P}(\mathrm{M})$ and $\mathrm{P}(\mathrm{R})$ became more stable and with increasing pattern. Therefore, higher confident in the results can be achieved with higher number of populations.

The effect of different crossover probabilities with a number of generation of 50 and a population size of 350 are considered in this case. Figure 4 shows the $\mathrm{P}(\mathrm{H}), \mathrm{P}(\mathrm{M})$ and $\mathrm{P}(\mathrm{R})$ obtained with the different crossover probabilities. Figures $4(\mathrm{a}$ and $\mathrm{b})$ show that the crossover probability does not have significant impact on the reliability indices $\mathrm{P}(\mathrm{H})$ and $\mathrm{P}(\mathrm{M})$. Figure 4(c) shows that the $\mathrm{P}(\mathrm{R})$ obtained using Equation 11 varies with the different values of the crossover probabilities. This variation was not caused by the crossover probabilities. This is due to the low probabilities of the risk states which cannot be recovered by the GA efficiently as explained earlier. $\mathrm{P}(\mathrm{R})$ obtained using Equation 12 shows no reaction to the variation in the crossover probabilities.

The effect of different mutation probabilities with a number of generation of 50 and a population size of 350 are considered in this case. A similar conclusion to that reached with the crossover probability is reached in this case. It is found that the mutation probability does not have a significant impact on the reliability indices. There was no variations in the $\mathrm{P}(\mathrm{H})$ and $\mathrm{P}(\mathrm{M})$ with the different mutation probability considered. However, there were some variations in the $\mathrm{P}(\mathrm{R})$ for the same reasons explained in the previous case with different crossover probability. 

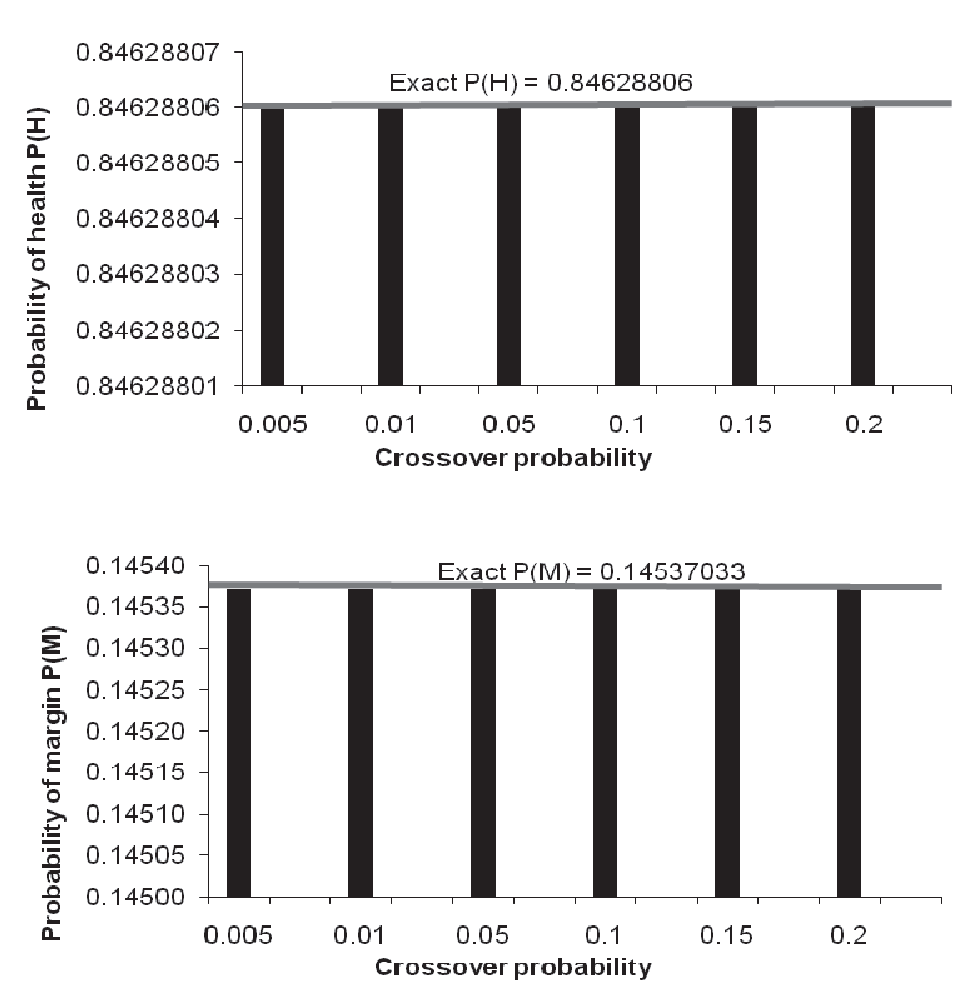

$\square \mathrm{P}(\mathrm{R})=\mathrm{Eq} \cdot 11 \mathrm{-P}(\mathrm{R})=\mathrm{Eq} \cdot 12$

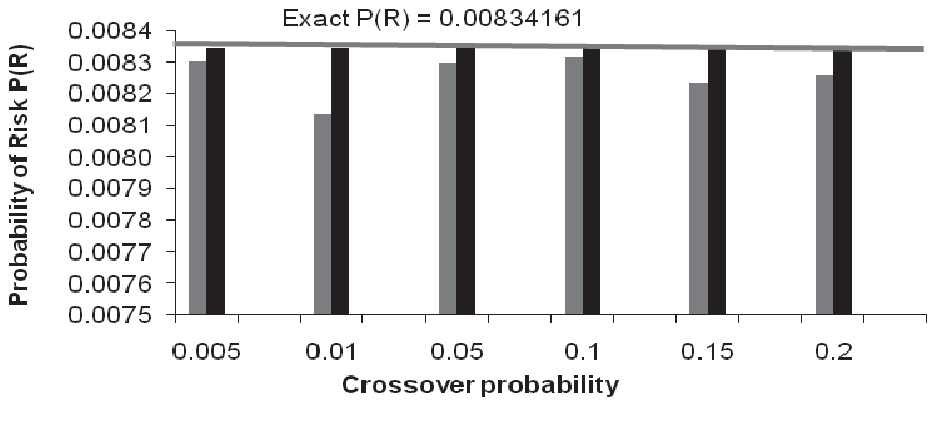

Fig. 4. Wellbeing indices with different crossover probabilities, generation size of 50 and population size of 350 .

The execution time for the above analysis was monitored and shown in Table 2. It can be seen that the running time increases with the increase in the number of generation and number of population. However, the increase in time is more aggressive with the increase in the number of generation. This is due to the fact that all GA operations are 
repeated with each and every new generation. Similar analysis was conducted on the crossover and mutation probabilities and it was found that no significant effect caused by the increase in these probabilities with respect to the execution time. This time is dominated mostly by the number of generation followed by number of population.

Table 2. Run time with respect to GA parameters.

\begin{tabular}{|ccccccccc}
\hline $\begin{array}{l}\text { Number of } \\
\text { generations }\end{array}$ & $\mathbf{1 0}$ & $\mathbf{2 0}$ & $\mathbf{3 0}$ & $\mathbf{4 0}$ & $\mathbf{5 0}$ & $\mathbf{1 0 0}$ & $\mathbf{2 5 0}$ & $\mathbf{5 0 0}$ \\
\hline $\begin{array}{l}\text { Run time } \\
\text { (Seconds) }\end{array}$ & 43 & 92 & 137 & 190 & 351 & 409 & 676 & 862 \\
\hline $\begin{array}{l}\text { Number of } \\
\text { population }\end{array}$ & $\mathbf{5 0}$ & $\mathbf{1 0 0}$ & $\mathbf{2 0 0}$ & $\mathbf{3 0 0}$ & $\mathbf{4 0 0}$ & $\mathbf{5 0 0}$ & $\mathbf{7 5 0}$ & $\mathbf{1 0 0 0}$ \\
\hline $\begin{array}{l}\text { Run time } \\
\text { (Seconds) }\end{array}$ & 261 & 288 & 317 & 335 & 392 & 522 & 641 & 711 \\
\hline
\end{tabular}

\section{Conclusions}

This paper presents a new GA based technique to calculate the annualized wellbeing indices for power generation systems. The features of the GA are utilized to identify the health, marginal and at risk wellbeing states and to calculate the associated wellbeing indices. The main feature of the proposed approach is that no complicated modeling is required unlike the case with the $\mathrm{CE}$ approach and the CPCOPT technique. Basic application to the IEEE-RBTS is considered. The results are validated with a conventional method. Sensitivity analysis is conducted to assess the effect of the GA parameters. The results illustrate that the $\mathrm{P}(\mathrm{M})$ reaches the exact value with higher number of generations and population sizes unlike the case with the $\mathrm{P}(\mathrm{H})$ in which the exact value is obtained with lower values. This is due to the fact that the $\mathrm{P}(\mathrm{H})$ is relatively high and the GA can easily recover the states with high probabilities. The $\mathrm{P}(\mathrm{M})$ requires higher number of generations and population sizes to recover the marginal states. It is also illustrated that the crossover probability has no significant effect on the calculated indices. It is found that no significant effect caused by the increase in the crossover or mutation probabilities with respect to the execution time. 
This time is dominated mostly by the number of generation followed by number of population.

This paper presents a basic GA search technique to calculate annualized wellbeing indices for the generation facilities using a constant load model. This technique can be extended in a future work to incorporate and investigate the effect of other factors such as variable loads and unit size and availability.

\section{References}

[1] Billinton, R. and Allan, R. N., "Reliability Evaluation of Power Systems", Plenum Press, New York (1996).

[2] Mori, H. and Kakuta, H., "A TS-based method for probabilistic reliability evaluation in power systems", Proceedings of the POWERCON 2010, Hangzhou, China, 2010, pp: 1-6.

[3] Skoonpong, A. and Sirisumrannukul, S., "Network reconfiguration for reliability worth enhancement in distribution systems by simulated annealing", 5th International Conference on Electrical Engineering/Electronics, Computer, Telecommunications and Information Technology, Krabi, Thailand, 2008, pp: $937-940$.

[4] Fangxing Li, "Distributed processing of reliability index assessment and reliability-based network reconfiguration in power distribution systems", IEEE Transactions on Power Systems, 20(1): 230-238, Feb. (2005).

[5] Billinton, R. and Fotuhi-Firuzabad, M., "Reserve Capacity Assessment in Small Isolated Electric Power Generating Systems", IEE Power Eng. Journal, 10(2): 73-80(1996).

[6] Billinton, R. and Karki, R., "Capacity Reserve Assessment Using System Wellbeing Analysis", IEEE Transactions on Power Systems, 14(2): 433-438, May (1999).

[7] Abdulwhab, A. and R. Billinton, "Generating System Wellbeing Index Evaluation", International Journal of Electric Power and Energy Systems, 26(3): 221-229, March (2004).

[8] Billinton, R. and Karki, R., "Application of Monte Carlo Simulation to Generating System Well-being Analysis", IEEE Transactions on Power Systems, 14(3) August (1999).

[9] Billinton, R., Karki, R. and Fotuhi-Firuzabad, M., "Probabilistic Methods Applied to Small Isolated Power Generating Systems", Proceedings of the 5th PMAPS International Conference, Vancouver, Canada, Sept., 1997, pp: 457-462.

[10] Al-Abdulwahab, A.S., "Incorporating Generating Unit Maintenance Scheduling in Power System Reliability", VDM \& Co. Publisher, Germany (2008).

[11] Abdulwhab, A.S. "Incorporating Generating Unit Maintenance Scheduling in Power System Reliability Evaluation", $P h$ D. Thesis, University of Saskatchewan, Saskatoon, Canada, (2003).

[12] Khatod, D. K., Pant, V. and Sharma, J. "Analytical Approach for Well-Being Assessment of Small Autonomous Power Systems With Solar and Wind Energy Sources", IEEE Transactions on Energy Conversion, 25(2) June (2010).

[13] Abdulwhab, A.S., Billinton, R., Eldamaty, A. A. and Faried, S. O., "Maintenance Scheduling Using a Genetic Algorithm (GA) with a probabilistic Fitness Function", Journal of Electric Power Components and Systems, 32(12) December: 1239-1254 (2004).

[14] Goldberg, D. E., "Genetic Algorithms in Search, Optimization and Machine Learning", Addison-Wesley Publishing company (1989). 
[15] Samaan, N. and Singh, C., "Using of Genetic Algorithms to Evaluate Frequency and Duration Indices for Generation System Reliability," Proc. ISAP 2001 Intelligent System Application to Power System Conf., Budapest, Hungary, pp:251-256.

[16] Samaan, N. and Singh, C., "Adequacy assessment of power system generation using a modified simple genetic algorithm", IEEE Trans. on PS, 17(4) November: 974-981 (2002).

[17] Masoum, M. A. S., Ladjevardi, M., Jafarian, A. and Fuchs, E. F., "Optimal Placement, Replacement and Sizing of Capacitor Banks in Distorted Distribution Networks by Genetic Algorithms", IEEE Transactions on Power Delivery, 19(4) October (2004).

[18] Todorovski, M. and Dragoslav, "A Power Flow Method Suitable For Solving OPF Problems Using Genetic Algorithms", EUROCON 2003 Ljubljana, Slovenia.

[19] Hui, Li, Li, Han, Bei, He and Shunchang, Yang, "Application Research Based on Improved Genetic Algorithm for Optimum Design of Power Transformers", Electrical Machines and Systems, Proceedings of the Fifth International Conference on Electrical Machines and Systems, 18-20 Aug. 2001, 242 - 245 , Vol. 1.,

[20] Rivas-Davalos, F. and Irving, M. R., "An Efficient Genetic Algorithm For Optimal LargeScale Power Distribution Network Planning”, 2003 IEEE Bologna Power Tech Conference, June 23-26, Bologna, Italy.

[21] Billinton, R., Kumar, S., Chowdhury, K., Chu, K., Debnath, K., Goel, L., Khan, E., Kos, P., Nourbakhsh, G. and Oteng-Adjei, J., "A Reliability Test System for Educational Purposes - Basic Data", IEEE Transactions on Power Systems, 4(3) August: 1238-1244 (1989). 


\section{تقييم موثوقية نظم توليد الطاقة الكهربائية باستخدام الخوارزمية الوراثية}

\section{أحمد صالح العبدالوهاب}

قسم الهندسة الكهربائية وهندة الحاسبات، كلية الهندسة، جامعة الملك عبدالعزيز،

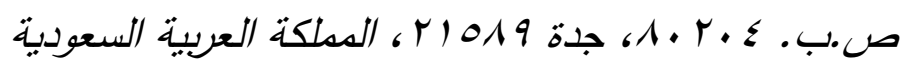

الكستخلص. جرت العادة على أن يتم تقيبم موثوقيـة نظم الطاقة

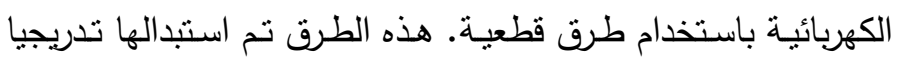

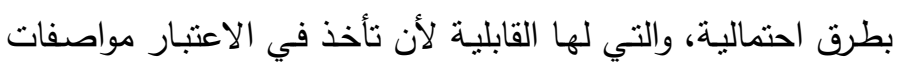

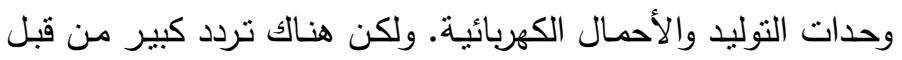
شـركات الكهربـاء لأن تستبعد الطرق القطعيـة. لذلك تلك تم استحداث

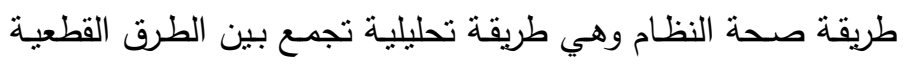

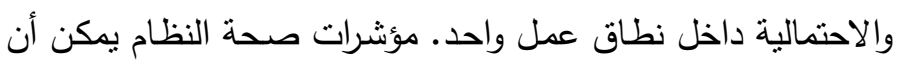

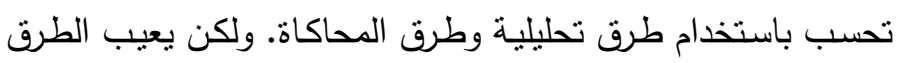

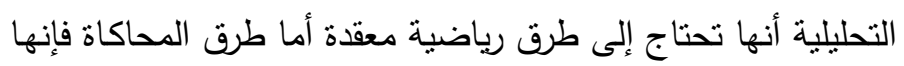

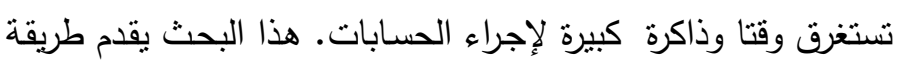

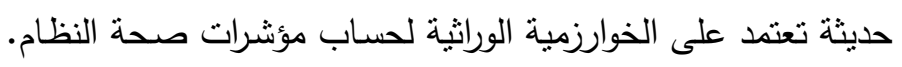

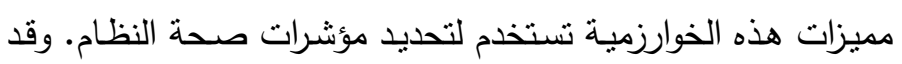

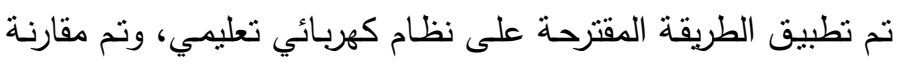

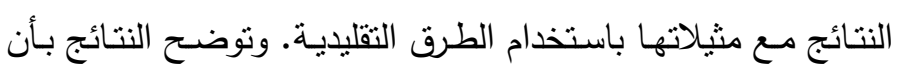

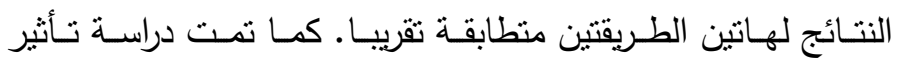

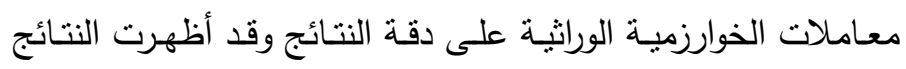
مدى سهولة الطريقة المقترحة. 